\title{
Computational Investigation of Flows in Diffusing S-shaped Intakes
}

R. Menzies

\begin{abstract}
This paper examines the flow in a diffusing s-shaped aircraft air intake using computational fluid dynamics (CFD) simulations. Diffusing s-shaped ducts such as the RAE intake model 2129 (M2129) give rise to complex flow patterns that develop as a result of the offset between the intake cowl plane and engine face plane. Euler results compare favourably with experiment and previous calculations for a low mass flow case. For a high mass flow case a converged steady solution was not found and the problem was then simulated using an unsteady flow solver. A choked flow at the intake throat and complex shock reflection system, together with a highly unsteady flow downstream of the first bend, yielded results that did not compare well with previous experimental data. Previous work had also experienced this problem and a modification to the geometry to account for flow separation was required to obtain a steady flow.

RANS results utilising a selection of turbulence models were more satisfactory. The low mass flow case showed good comparison with experiment and previous calculations. A problem of the low mass flow case is the prediction of secondary flow. It was found that the SST turbulence model best predicted this feature. Fully converged high mass flow results were obtained. Once more, SST results proved to match experiment and previous computations the best. Problems with the prediction of the flow in the cowl region of the duct were experienced with the $S$-A and $k$ - $\omega$ models. One of the main problems of turbulence closures in intake flows is the transition of the freestream from laminar to turbulent over the intake cowl region. It is likely that the improvement in this prediction using the SST turbulence model will lead to more satisfactory results for both high and low mass flow rates.
\end{abstract}

Keywords: aerodynamics, computational fluid dynamics, internal flows, turbulence models.

\section{Introduction}

For over three decades the study of air intakes has led to design improvements based on wind tunnel test data. Problems with particular designs such as damage to intake structures as a result of engine surge tended to only be found after prototype testing. From the early 1970's wind tunnel testing methods improved considerably and there was also a much greater understanding of some important characteristics of duct flows. During this time CFD techniques have become widely used and advances have led to ever more complex studies yielding excellent agreement with experimental data. CFD methods are advantageous as they are generally cheaper in terms of cost, time and resources. However $C F D$ should be thought of as an aid to experimental work. The validation of computational results with previous work and experimental data is crucial and is the motivation for this paper.

Air intakes are a vital component of aircraft and the primary purpose is to offer the engine a uniform stream of air. The efficiency of such devices is crucial in that it makes a contribution to the handling characteristics and performance attributes of the aircraft. Just as important is the need for engine/intake compatibility. Engine surge can be induced if factors such as cowl lip shape and diffuser shape are not closely considered in the design process. The design of an aircraft intake will depend on the role of the aircraft and the conditions in which it operates. Subsonic intakes tend to be shorter in length due to the lower speeds when compared with a supersonic intake. However the position of store bays/undercarriage wells or the need to mask the compressor face in order to reduce the radar cross section and hence observability of the aircraft can lead to offset intakes such as the M2129.

The flow in diffusing s-ducts is complex in nature due to effects arising from the offset between the intake plane and engine face plane. As the flow enters the intake it accelerates and then meets the first bend of the intake, where the centrifugal and pressure forces acting on the faster moving core cause it to move towards the outside of the bend (port side), where there is an adverse pressure region. Near wall fluid that is energy deficient cannot pass through the adverse pressure gradient. Instead the flow moves around the outer walls towards the region of low static pressure on the inside of the bend. This feature sets up two cells of swirling secondary flow. As the flow moves on through the duct it would perhaps be expected that a similar motion in the opposite sense be initiated at the second bend. However by this stage the low energy flow is largely on the outside wall relative to the second bend and is not driven back circumferentially. Thus the swirl experienced at the engine face is in the sense generated as a result of the first bend.

Engine/intake compatibility is purely concerned with the quality of the airflow that is delivered by the intake to the compressor face and how the engine is affected. The flow distribution across the compressor face should be as uniform as possible to maximise performance of the engine and reduce the possibility of undesirable occurrences such as engine surge. Total elimination of non-uniformity in pressure across the compressor face is not possible but the degree can be minimised. Distortion is the term used to describe poor pressure distribution, and strong secondary flow causes poor distortion and can be sufficient to induce surge and produce a propagating hammershock. 


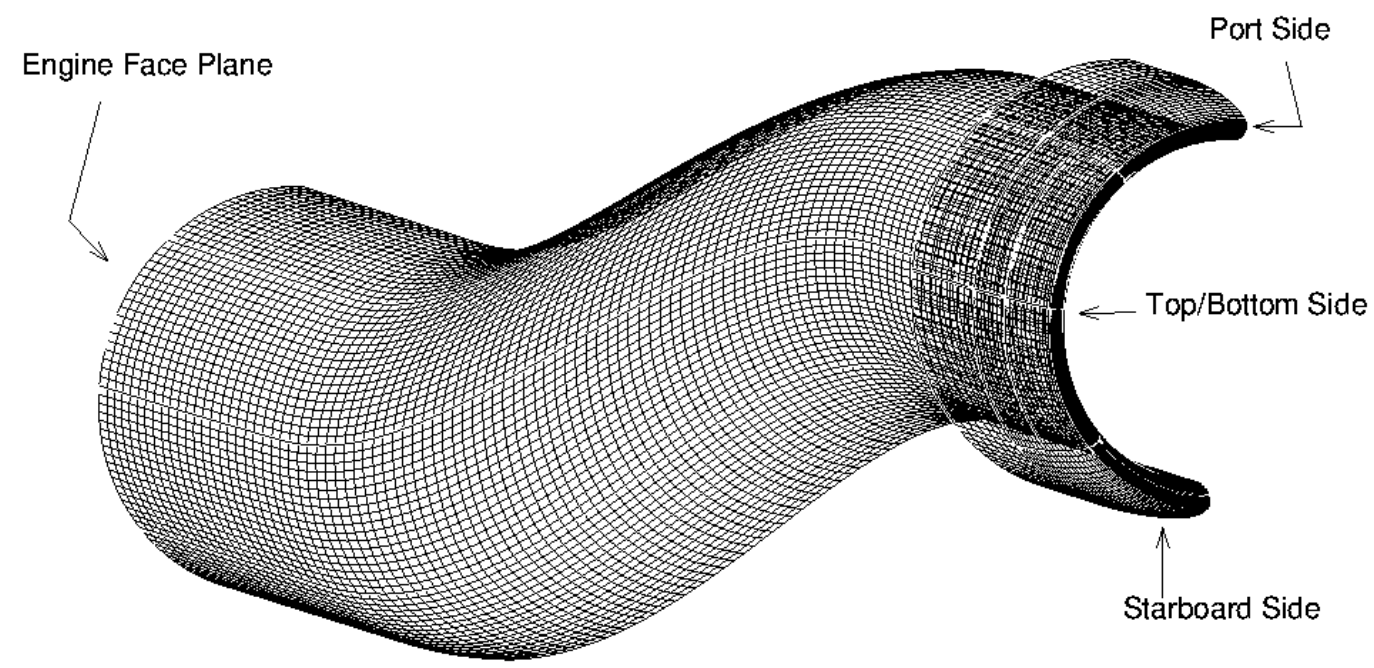

Fig. 1: M2129 showing data line extraction definitions

The geometry of the intake is shown in Figure 1. Extracted data in this paper used for comparisons is taken from the port and starboard side of the intake.

\section{Numerical method}

The flow solver used for the calculations was the University of Glasgow's three dimensional flow solver named $P M B 3 D$. It has previously been applied to a number of problems including:

- Inviscid and turbulent wings,

- Inviscid, laminar and turbulent ogive cylinders at incidence,

- Cavity flows,

- Rolling, pitching, and yawing delta wings,

- Other complex three dimensional geometries.

A cell centred finite volume technique is used to solve Euler and the Reynolds averaged Navier-Stokes (RANS) equations. The diffusive terms are discretised using a central differencing scheme and the convective terms use Roe's scheme with MUSCL interpolation offering third order accuracy. Steady and unsteady flows can be solved. Steady flow calculations proceed in two parts, initially running an explicit scheme to smooth out the flow at a small CFL and then switching to an implicit algorithm to obtain rapid convergence. The preconditioning is based on Block Incomplete Lower-Upper (BILU) factorisation which is also decoupled between blocks to help reduce computational time. The linear system arising at each implicit step is solved using a Generalised Conjugate Gradient (GCG) method. The unsteady code uses an implicit unfactored dual time approach and the rate of convergence between the two consecutive time steps is monitored by the Pseudo Time Tolerance (PTT). More information can be found in reference [1].

The RANS calculations were run using Spalart-Allma-

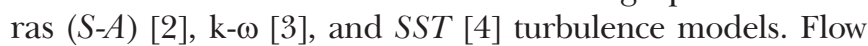
separation and large secondary flows due to adverse pressure gradients generated by localised accelerating and decelerating flows create high demands on turbulence models. The $S-A$ model is of the one-equation type and is generated from first principles. These models are satisfactory and have been shown to be as successful as mixing length models. However a more universal model is desirable, particularly for separated flows. The $k-\omega$ model is based on a two-equation approach which has served as the foundation for much of the turbulence model research over the past two decades. This model accounts for the computation of the turbulent kinetic energy $(k)$ but also for the turbulent length scale. Consequently two-equation models can be used to predict properties of a given flow without any prior knowledge of the turbulent structure. The Shear Stress Transport (SST) model is a modified version of the $k$ - $\omega$ model and is designed to account for the transport of the principal turbulent stress. The modifications improve the prediction of flows with strong adverse pressure gradients and separation and hence the $S S T$ turbulence model is thought to be more suitable to the application of internal duct flows, as studied here.

A modification had to be made to the existing boundary conditions in $P M B 3 D$ to account for the simulation of the engine face. Simple extrapolation is used with the exception of static pressure which is held constant. Although small amounts of bulk swirl have been shown to impose back into the main flow, the effects have been proven to be negligible experimentally and thus this method of modelling the engine face is satisfactory. The value of the constant static pressure set at the engine face depends on the engine demand that is to be modelled, and is determined from the freestream Mach number $(M)$, the contraction ratio (ratio of the intake plane area to engine face plane area), the desired pressure ratio and mass flow rate (used in wind tunnel tests).

The multiblock grid was generated using the commercial package ICEMCFD. An extensive farfield region is included upstream of the intake in order to allow for the direct comparison of results between different flow solvers (intake entry conditions need not be specified as the flow is entering from freestream conditions). The interaction of these freestream blocks with the intake, particularly the cowl fold back, leads to some complex topologies. In summary, an O-grid is used in the intake. The outer blocks of the O-grid are then wrapped around at the intake entry plane to form a C-grid for the intake cowl. This then allows 
an $\mathrm{H}$-grid to be used in the large farfield region which has the advantage of reducing grid size in regions in which the flow is at freestream conditions.

The RANS investigation of complex three-dimensional flows inevitability involve the use of dense grids. The computational fluid dynamics group at the University of Glasgow owns a cluster of high-tech PC's allowing demanding cases to be studied. The cluster consists of 32 nodes, each a $750 \mathrm{MHz}$ AMD Athlon thunderbird uni-processor machine with $768 \mathrm{Mb}$ of $100 \mathrm{MHz}$ DRAM.

\section{Results}

The low mass flow rate (LMFR) case simulates low engine demand and is the simpler of the two test cases. Euler and RANS calculations were examined and compared with previous computations and experimental data [5]. A typical fully converged turbulent calculation required around $2000 \mathrm{im}-$ plicit steps at a CFL of 30 for a medium sized grid of around 400,000 points. This took about 6 hours of computational time using 8 processors. Euler and RANS results were also computed for the high mass flow case. However this case is more complicated as supersonic flow is generated as the freestream is accelerated into the duct. Supersonic flow is also generated as the flow accelerates around the first bend of the intake. This leads to an unrealistic unsteady flow predicted by the Euler calculations. Problems have been encountered in previous work with this case [6]. A resolution was found by modifying the geometry on the starboard side following the first bend to account for flow separation. This modification was not implemented in this work with attention instead focusing on the RANS results.

\subsection{Low mass flow case}

The low mass flow rate $(L M F R)$ case is the simpler of the two cases and the Euler solution provides a good introduction to the problem and is straightforward to study. Comparison of the results with RANS computations and experiment data can be seen in Figures 2 and 3. Local static pressure $\left(P_{\mathrm{s}}\right)$ is non-dimensionalised with total freestream pressure $\left(P_{\mathrm{T}}\right)$ and is plotted against duct length, $X$ (non-dimensionalised with engine face diameter, $D$ ). Reasonable agreement of the flow with computation is seen. The location of the stagnation point is well predicted. However upstream of the first bend $(X / D=1)$ in the cowl region there are large differences with experimental data. Flow acceleration from the stagnation point peak pressure to a minimum just inside the cowl interior is over-predicted. This leads under-prediction in the pressure recovery after the duct throat. Consequently flow acceleration is also over-predicted at the second bend starboard side (as shown in Figure 1 at $X / D=1$ ). It is thought that these differences may be because of viscous effects that are being neglected in the Euler calculation.

The RANS calculations reduce the magnitude of the over and under predictions in pressure, caused by the flow accelerating from stagnation into the intake, for all turbulence models examined. The RANS calculations used a Reynolds number $(R e)$ of 777,000 based on the engine face diameter. The peak pressure (stagnation point) on the outside of the cowl wall is well matched. The flow generated through the first bend of the intake is also better predicted. The $k-\omega$ results appear to match experimental data the best in the cowl region. SST and $S$ - $A$ results are very similar in this area. Downstream of the first bend the port and starboard side data comparisons differ. Port extractions match fairly well with experimental data although $S A$ and SST results are consistently higher, probably due to the slight over prediction in the pressure recovery after the initial acceleration into the duct.

However the most interesting examination is from the starboard side. A feature of s-duct intake flow is the generation of secondary flow off the first bend as described in the introduction. The extent of the secondary flow for the low mass flow case is indicated by a pressure drop between the two bends of the duct (between an X/D of 1 and 3.5). Figure 2 shows that all turbulence models fail to predict the drop witnessed in the experiments. However closer examination shows that the $S-A$ and, more particularly, the SST models show a slight levelling off of the pressure which is indicative of secondary flow development. The extent of the drop is not

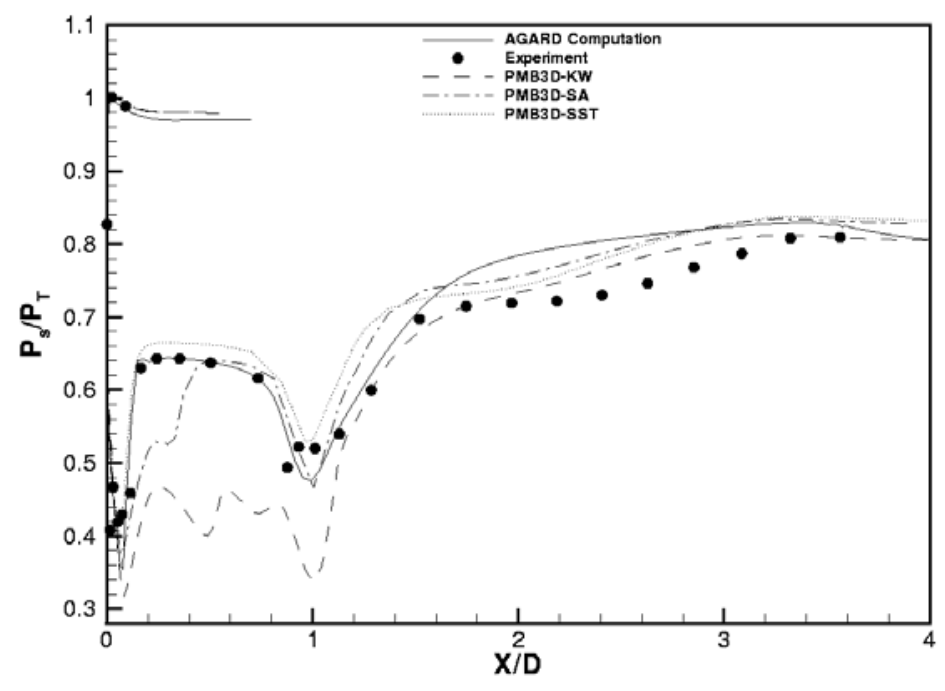

Fig. 2: LMFR calculation - data extracted from starboard side of intake 


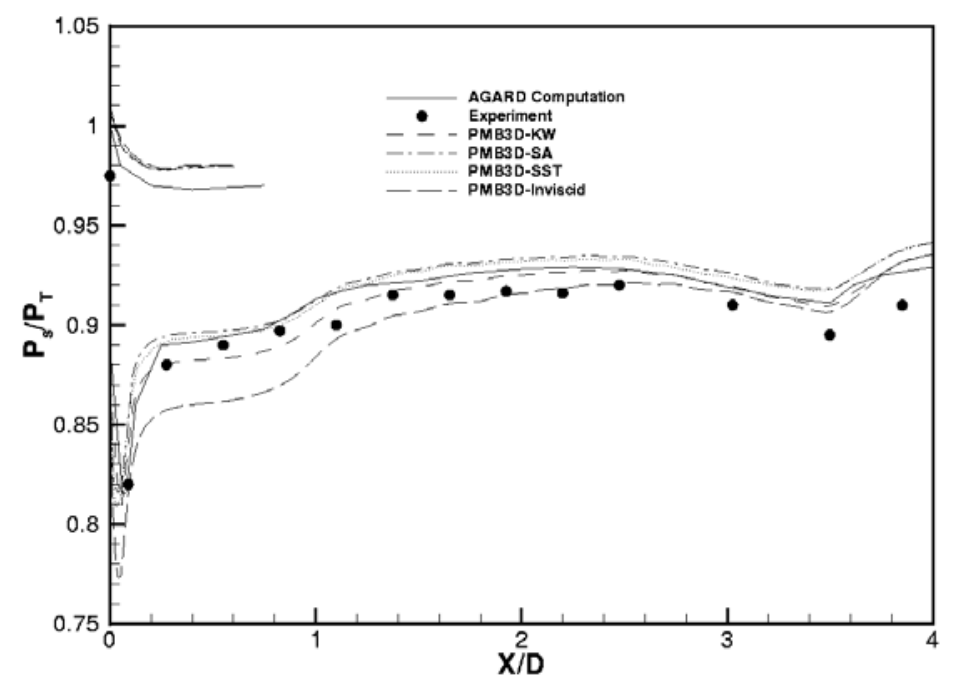

Fig. 3: LMFR calculation - data extracted from port side of intake

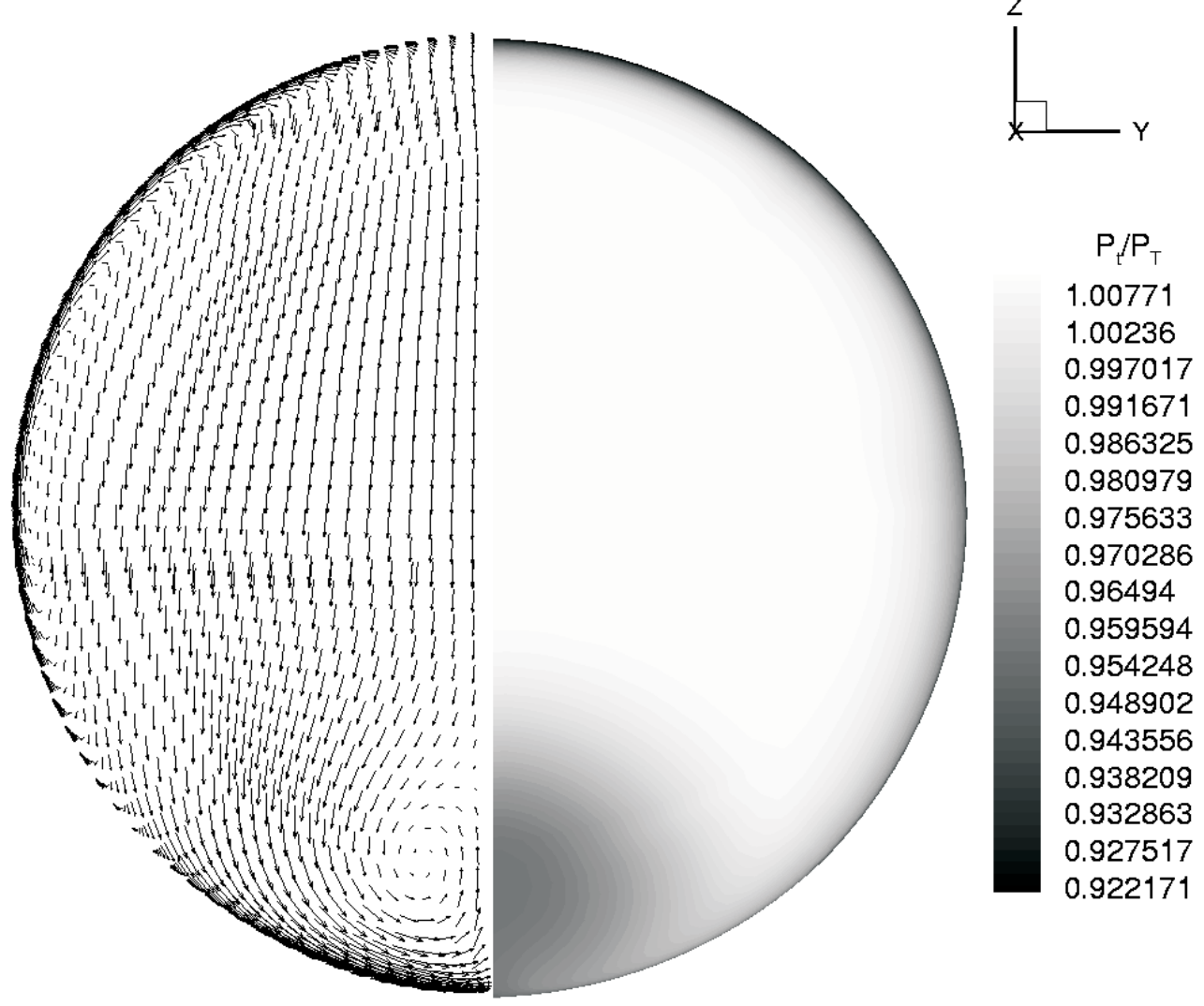

Fig. 4: LMFR N-S SST calculation - secondary flow at engine face

well matched with experiment but it should be remembered that these models have a higher pressure recovery than expected which could explain this. The secondary flow can be seen in Figure 4 by means of velocity vectors and local total pressure contours (non-dimensionalised with freestream total pressure) for the SST model.

\subsection{High mass flow case}

The high mass flow rate $(H M F R)$ case is more difficult to model due to the complex nature of the flow generated. Supersonic flow is achieved as the flow accelerates into the intake and also as the flow accelerates around the starboard 
side first bend. Fully converged solutions were obtained for coarse, medium, and fine grids with the residuals dropping by 8 orders of magnitude. The results using the various turbulence models were compared with previous computations and experimental data.

As with the LMFR calculations, the Euler calculations were qualitatively in agreement with previous works but quantitatively showed differences, since no allowances were made to account for boundary layers and separation in the inviscid calculations.

Fully converged viscous results were achieved using all turbulence models. Figures 5 and 6 show extracted pressures from the starboard and port sides respectively. It is immediately obvious that there are problems in the cowl region with the $k-\omega$ and $S-A$ results. Following the initial pressure drop resulting from the flow acceleration into the duct, further drops occur, especially for the $k-\omega$ model. Examining an extraction through the symmetry plane of the grid shows a complex shock wave reflection system, contrary to experi- ment. This appears to stem from an acceleration into the duct that is greater than in experiment leading to higher core Mach numbers. Consequently the pressure never recovers prior to the first bend. After the first bend the pressure recovers to match previous solutions and experiment well.

The SST model matches experiment very well. The acceleration of the flow into the duct compares very closely with experiment. The subsequent pressure recovery and flow acceleration around the starboard side first bend also match very well. The reason for the superior prediction using the SST model appears to stem from the cowl region and a better prediction of the initial flow features. Closer examination of the solution shows that the transition from freestream to turbulent conditions was different from the other models. The $S$ - $A$ model also suffers from an over acceleration of the flow into the duct but the extent of this is not as large as for the $k$ - $\omega$ model and the flow recovers prior to the first bend.

The secondary flow developed in the intake duct can be seen in Figure 5 in the form of a levelling of the pressure trace

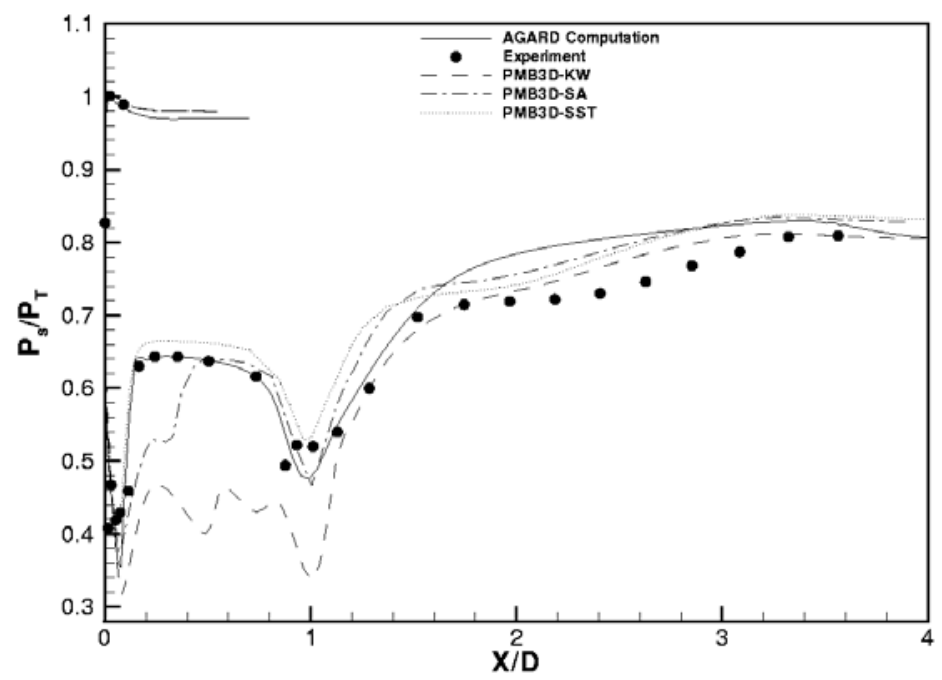

Fig. 5: HMFR calculation - data extracted from starboard side of intake

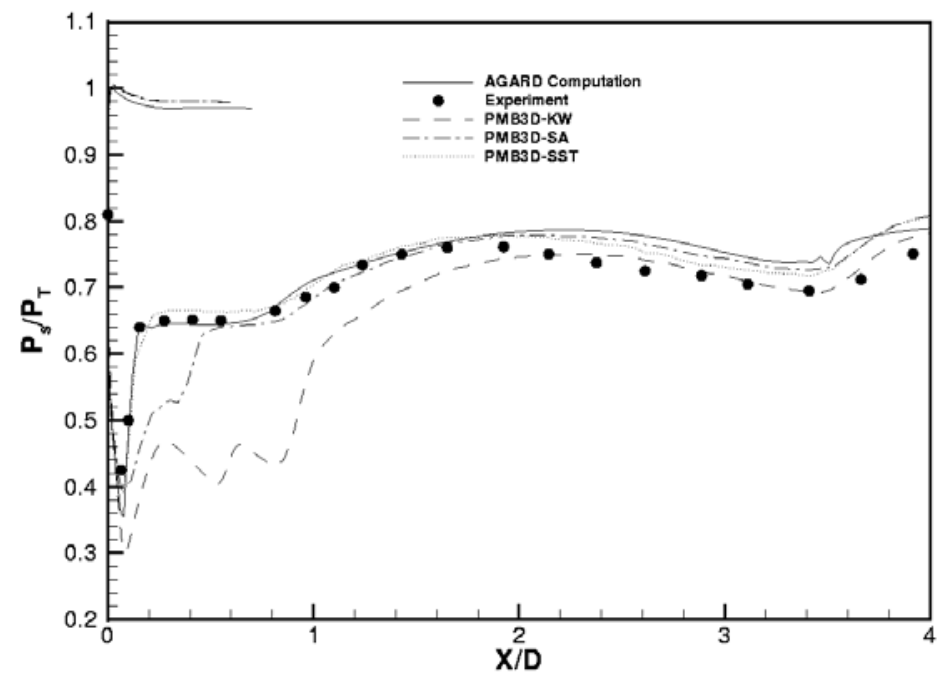

Fig. 6: HMFR calculation - data extracted from port side of intake 


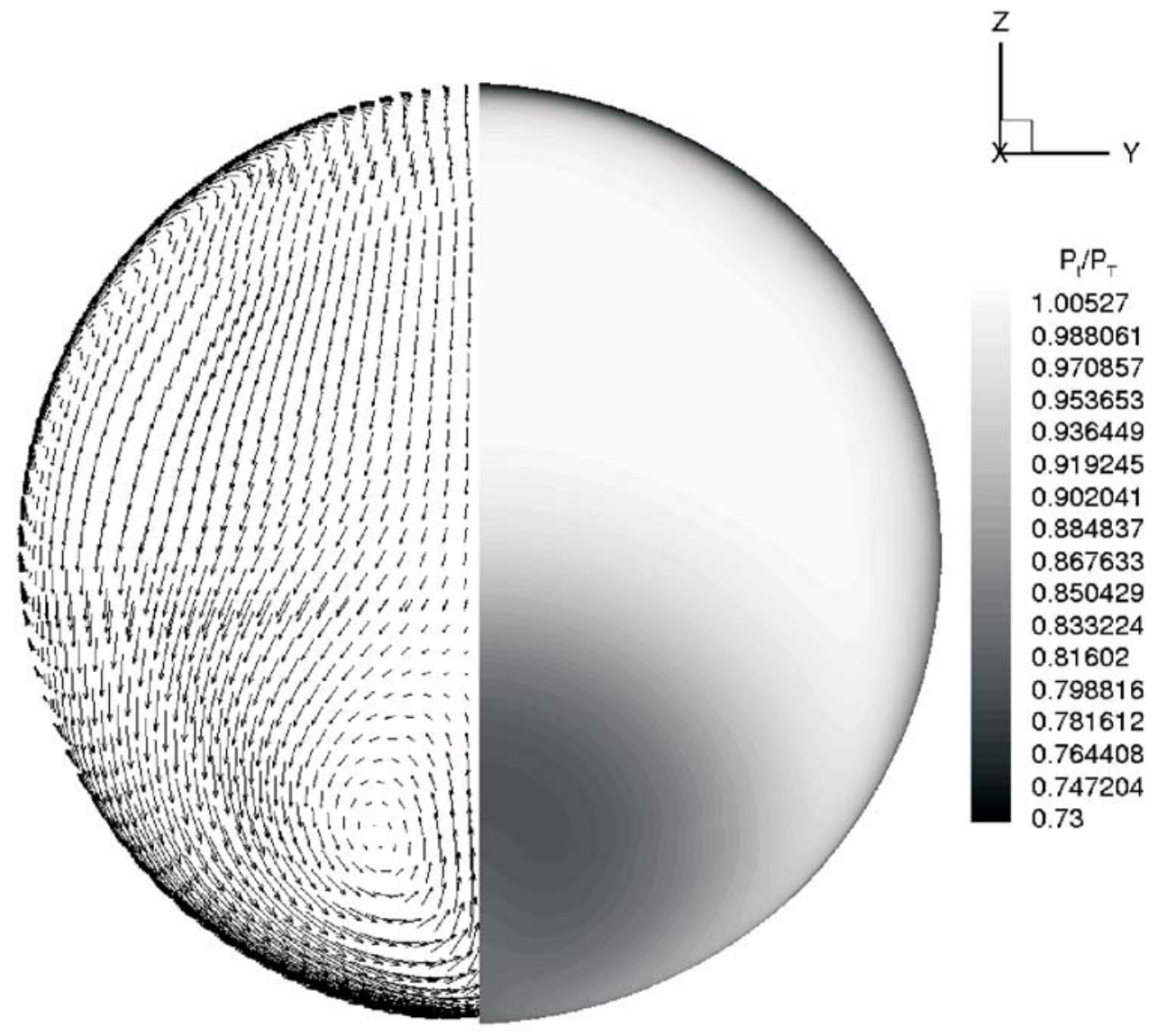

Fig. 7: HMFR N-S SST calculation - secondary flow at engine face

between the two bends. Closer examination of Figure 5 shows that the SST model again predicts the secondary flow better than the other models. Figure 7 shows the effect of the secondary flow at the engine face. It is clear that the secondary flow developed is significant and it is not untenable to suggest that the level of distortion experienced at the engine face might induce compressor blade stalling and subsequent engine surge.

\section{Conclusions}

Euler and RANS calculations have been performed on an offset, diffusing intake duct. A variety of turbulence models were used with the aim of validating the flow. Two separate cases were looked at. Firstly, a low mass flow case to simulate low engine demand, and, secondly, a high mass flow case to simulate high engine demand.

Comparisons were made by examining the local static pressure histories through the duct (non-dimensionalised with freestream total pressure). Euler calculation were initially done as they are straightforward and serve as a good introduction to the problem. Low mass flow results showed that, qualitatively, the salient flow features are captured, but quantitatively there were over-predictions in the level of acceleration into the duct leading to large pressure drops and poor pressure recoveries. High mass flow Euler results failed to converge. The case is complex as supersonic flow is generated as the flow accelerates into the duct, and also as the flow accelerates around the starboard side first bend. It was found that the solution is unsteady, with the unsteadiness originating from the starboard side first bend. Experimental data shows that there is considerable separation from this location and the Euler code cannot predict this without modifications being made to the geometry.

$R A N S$ results were computed using $S-A, k-\omega$, and $S S T$ turbulence models. Fully converged steady solutions were reached. The low mass flow case showed that the SST model performed more satisfactorily than the other models. Flow acceleration was closely matched into the duct although the subsequent pressure recovery was over predicted. This led to a lower acceleration around the first intake bend than was witnessed in the experiment. One of the main challenges with the low mass flow case is the difficulty of predicting secondary flow. However there is evidence that the SST model (and also the $S$ - $A$ model) predict limited secondary flow as indicated by a levelling of the pressure trace on the starboard side, although the actual pressure magnitude is too high due to the reduced acceleration around the intake first bend.

Results from the $k$ - $\omega$ turbulence model for the high mass flow case showed that the acceleration into the duct was over predicted leading to an significant supersonic flow in the cowl region. There is evidence of shock reflection and the flow can be described as choked. This is all contrary to experiment. Similar results can be seen for the $S$ - $A$ model although the solution is generally better. The $S S T$ model once again provides the best comparison with experiment. The level of acceleration into the duct is well predicted although, as for the low mass flow case, the pressure recovery is a little better 
than witnessed in experiment. Secondary flow on the starboard side following the first bend is predicted for all turbulence models although the SST model predicts the feature best.

This work has served as a validation of the application of $P M B 3 D$ to problems of this type. It has been found that the SST turbulence model appears to offer the best overall results to this type of problem. A non-algebraic model [7] will shortly be tested and it is anticipated that this will provide further improvements.

\section{Acknowledgements}

The author gratefully thanks K. J. Badcock and B. E. Richards in the Department of Aerospace Engineering and M. Jackson at DERA Bedford for their help. Thanks also to other members of the computational fluid dynamics group in the department for their suggestions and assistance. This work is supported by sponsorship from DERA Bedford.

\section{References}

[1] Badcock, K. J., Richards, B. E., Woodgate, M. A.: Elements of Computational Fluid Dynamics on Block Structured Grids Using Implicit Flow Solvers. Progress in Aerospace Sciences, Vol. 36, 2000, pp. 351-392

[2] Spalart, P. R., Allmaras, S. R.: A One-equation Turbulence Model for Aerodynamic Flows. AIAA, Paper 92-0439, 1992
[3] Wilcox, D. C.: Turbulence Modelling for CFD. DCW Industries, Inc., La Canada, California, 1994

[4] Menter, F. R.: Zonal Two Equation Kappa-Omega Turbulence Models for Aerodynamic Flows. AIAA, Paper 93-2906, 1993

[5] Fluid Dynamics Panel Working Group 13: Test Case 3 Subsonic/Transonic Circular Intake. AGARD Advisory Report 270, 1991

[6] May, N. E., McHugh, C. A., Peace, A. J.: An Investigation of Two Intake/S-Bend Diffuser Geometries Using the Sauna CFD System - Phase 1. Aircraft Research Association, MEMO 386, 1993

[7] Craft, T. J., Launder, B. E., Suga, K.: Development and Application of a Cubic Eddy-Viscosity Model of Turbulence. International Journal of Heat and Fluid Flow, 17, 1996, pp. 108-115

Ryan D. D. Menzies, M.Eng.

phone: +4401413302227

fax: +4401413305560

e-mail: rmenzies@aero.gla.ac.uk

Department of Aerospace Engineering

James Watt Building (south)

University of Glasgow

Glasgow, G12 8QQ

Scotland, UK 